\title{
Addressing stigma and gender inequities to strengthen HIV and AIDS programming: A focus on key populations
}

Population Council

Follow this and additional works at: https://knowledgecommons.popcouncil.org/departments_sbsr-hiv

Part of the Demography, Population, and Ecology Commons, Family, Life Course, and Society Commons, and the International Public Health Commons How does access to this work benefit you? Let us know!

\section{Recommended Citation}

Population Council. 2018. "Addressing stigma and gender inequities to strengthen HIV and AIDS programming: A focus on key populations," fact sheet. Washington, DC: Population Council. 


\section{ADDRESSING STIGMA AND GENDER INEQUITIES TO STRENGTHEN HIV AND AIDS PROGRAMMING: A FOCUS ON KEY POPULATIONS}

Over the past 20 years, the Population Council has generated evidence around the types of stigma experienced by key populations at higher risk for HIV (KPs), and has designed and tested strategies aimed at reducing stigma and improving HIV programs and outcomes. KPs, such as female sex workers (FSWs), men who have sex with men (MSM), transgender individuals, and people who use drugs (PWUD), often face multiple stigmas that reinforce one another. These stigmas, which have been named intersecting, layered, or compounded stigmas, include HIV-related stigma, and stigma due to KP identities, behaviors and/or occupations. Addressing stigma has been a priority for the Population Council both because it is a human rights violation and a major barrier to a successful HIV response. This brief summarizes Population Council findings on KPs' experiences of stigma and strategies tested to reduce the stigma and discrimination KPs face.

\section{HOW IS STIGMA EXPERIENCED BY MEMBERS OF DIFFERENT KEY POPULATIONS?}

\section{KPs experience both external stigma and internalized stigma which can raise HIV risk.}

Early Council research documented high levels of KP stigma and discrimination, such as physical and emotional abuse, denial of services, and rejection by support networks. Moreover, such stigma contributes to HIV risk. For example, findings in Zambia ${ }^{1}$, Kenya ${ }^{2}$, and India ${ }^{3}$ showed that sexual and physical abuse among FSWs and transgender sex workers from clients impedes their ability to control sexual transactions, including type of sex act, payment, condom use, and consensual sex practices. Additionally, research in South Africa ${ }^{4}$ among MSM found that recurrent discrimination can fuel internalized homophobia and feelings of shame, which are both correlated with higher levels of HIV misinformation and adoption of fewer HIV protective strategies.

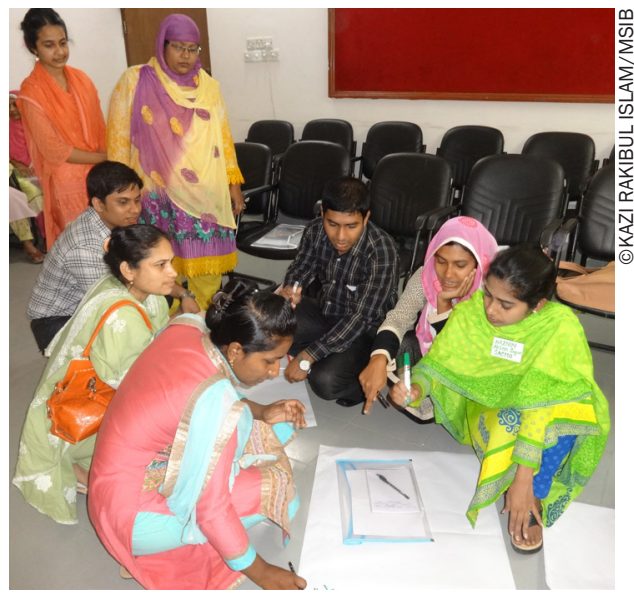

As part of Link Up, the Population Council evaluated a training program designed to reduce provider stigma toward young, marginalized clients. 


\section{KPs' often experience stigma in the healthcare setting.}

Findings from several countries documented early on discriminatory practices and stigmatizing attitudes of health workers both toward people living with HIV (PLHIV) and KPs. Research from India ${ }^{3}$, Zambia ${ }^{5}$, South Africa $^{6}$, and Senegal ${ }^{7}$ found that health providers were frequently unprepared to respond to the needs of KPs living with HIV, and engaged in practices such as labelling beds of those living with HIV or refusing to offer services.

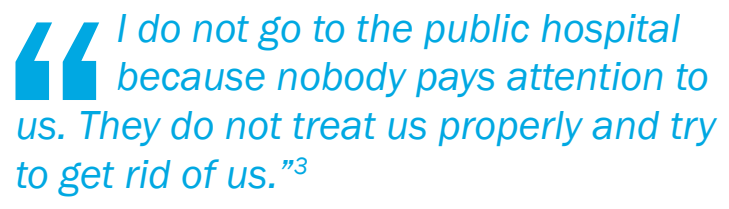

4 Health workers do not understand what our needs are. They are not adequately trained to provide services to LGBTI."6

\section{KP stigma is driven by both fear and negative value judgements.}

Evidence suggests that stigma is based both on fear of transmission and negative value judgements associated with behaviors and identities, so both should be addressed to successfully reduce stigma. Findings from Nicaragua ${ }^{8}$ and Senegal ${ }^{7}$ show that KPs are frequently blamed for their own HIV status when positive. Furthermore, healthcare providers in Vietnam may also stereotypically link KPs to HIV, due to their appearance or behavior?

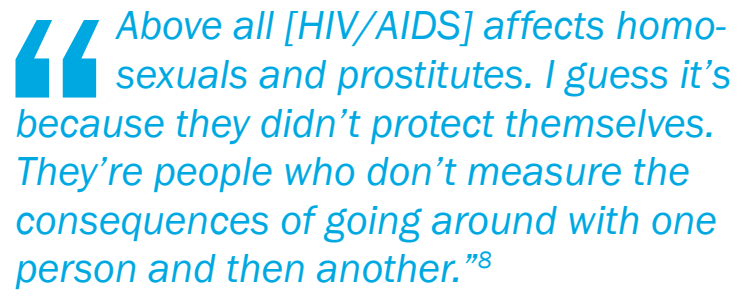

-Nicaragua

\section{Anticipated stigma inhibits successful service use.}

KPs regularly report avoiding healthcare services because of fear of encountering HIV-related stigma and stigmatizing attitudes related to their identities and practices. Findings from Nigeria ${ }^{9}$ and $Z^{2 a m b i a}{ }^{5}$ demonstrate that even among those who do access services, many avoid disclosing sexual practices or STI symptoms to providers as it may expose their KP status. Furthermore, research conducted in Myanmar ${ }^{10}$, Zambia $^{5}$, and Nigeria ${ }^{9}$ found that MSM fear that their sexual orientation or practices could be exposed if they are seen accessing sites for HIV testing or antiretroviral treatment (ART).

\section{In Nigeria the number one barrier has always been discrimination. You might go to a clinic or a hospital, and probably l'm faced with an STI that is MSM related, what will I say? How will I tell it to a doctor or a nurse that has been there?"9}

-Nigeria

\section{PROMISING INTERVENTION STRATEGIES TO REDUCE INTERSECTIONAL STIGMA}

While stigma is an experience common to members of all KPs, strategies to reduce stigma will depend upon the specific needs of individuals. The Council has helped design several stigma reduction programs as well as demonstrated their effectiveness, which can inform future program adoption and scale up.

\section{Training and sensitizing healthcare providers}

Evaluations of training programs in Bangladesh ${ }^{11}$, Vietnam ${ }^{7}$, and Senegal ${ }^{12}$ demonstrated significant reductions in providers' stigmatizing beliefs and reduced discriminatory actions while treating PLHIV. In Vietnam, for example, the number of hospital workers who felt that HIV/AIDS was a punishment for "bad behavior" was reduced by almost half.

Training providers can also raise client satisfaction after the intervention. In Bangladesh ${ }^{11}$, FSW and 
MSM respondents reported a substantial decrease of enacted stigma and discriminatory attitudes at healthcare services. An intervention in Senegal ${ }^{12}$ trained a network of health providers on the needs of MSM, and how to deliver high quality, confidential, non-stigmatizing STI services and psychosocial care. Findings showed that men valued the access to quality, non-judgmental testing and treatment services.

Before I joined [the] Link Up proj-
ect I used to think how am I going
to deal with transgender people and
MSM. I was kind of afraid, but when I
attended counselor training, I realized
that they are part of us. I should provide
service to them as they are part of our
society."11

-Bangladesh

\begin{abstract}
7 The medical officers are very kind and available to attend to us...if we had been mistreated, it is obvious that we would not have returned...W We receive counseling which is very vital to us because it is directly related to our activities"12
\end{abstract}

\section{Bringing HIV services to KPs in the community}

Given the low uptake of HIV services in mainstream healthcare settings by KPs due to stigma and criminalization, relatively new strategies such as HIV self-testing kits (HIVST) and community-based delivery of ART offer alternatives to facility-based government HIV services. A Council-led HIVST study in Nigeria ${ }^{13}$ that provided MSM with self-testing kits reported high levels of uptake and subsequent linkage to treatment among those who tested positive. And, emerging findings from an ongoing community-based ART distribution intervention in Tanzania ${ }^{14}$ for FSWs is showing positive results-higher ART initiation, retention, and adherence after six months among FSWs participating in the community-based ART model compared to standard facility-based ART provision.

\begin{abstract}
4 It was my actual first time of getting tested, I haven't gotten tested before....actually this stuff [HIV self-testing] is great and at least I got to test myself at my own convenient time and got to know my result before going through any other process." 13
\end{abstract}

-Nigeria

\begin{abstract}
4 They speak to us politely and don't stigmatize us. If they need to run tests, they touch my body normally without feeling disgusted.... They even take their time to call us, asking about our health progress which is really sweet."14
\end{abstract}

-Tanzania

\section{KP-sensitive community health centers}

In 2013 the Population Council established a community health center $(\mathrm{CHC})$ in Lagos with the objective of delivering comprehensive HIV prevention and treatment services for KPs, advocating for their health needs, and linking KPs to other inclusive services. The $\mathrm{CHC}$ is run with medical professionals trained in the needs of KPs and are committed to ensuring sensitive, friendly and non-stigmatizing high-quality services. In 2015, the $\mathrm{CHC}$ began a "hub-spoke" model, expanding its services to 15 small satellite facilities, run by the local government or by community-based organizations, to better reach geographically-distant KP individuals. The number of individuals who have been tested for HIV (and received their results) through the $\mathrm{CHC}$ has risen from 1,338 in 2013 to 18,492 in $2017^{15}$.

\section{Mass communication strategies}

The Council evaluated an intervention led by Puntos de Encuentro in Nicaragua, which consisted of community-based activities as well as education and entertainment programming, including a national television soap opera (Sexto Sentido) aimed at youth. Results showed that individuals who were exposed to the material-particularly the soap operawere less likely to have negative views of PLHIV and homosexuality, and reduced the perception that KPs were responsible for HIV. Some aspects of stigma changed more than others, however, as 
stigma exclusively toward KPs was more difficult to reduce than that related to HIV alone. This further demonstrates the complexities when attempting to address intersecting stigmas ${ }^{8}$.

\section{Before the [Sexto Sentido] series, people didn't think that gays and lesbians thought the same way as us, but now I know they're human. I feel great admiration for gays; they're brave. I used to find them repugnant; but not now."}

\section{MEASURING LAYERED/ COMPOUNDED STIGMA}

One of the challenges in addressing layered/

compounded stigma has been the lack of standardized, quantitative tools to effectively measure it. The People Living with HIV Stigma Index (Stigma Index) has been the most widely used measure to capture stigma from the perspective of PLHIV. The Population Council worked with the Stigma Index developers to create an updated version (Stigma Index 2.0) that more fully captures intersecting stigmas. The instrument was piloted in Uganda, Cameroon, and Senegal, reaching over 1,200 PLHIV demonstrating that use of the measurement and empowerment tool can inform programs and policies in addressing intersecting stigmas ${ }^{16}$.

\section{\#\#\#}

Evidence generated by the Population Council has shown that addressing and reducing stigma faced by KPs is a crucial step toward improving HIV prevention and treatment outcomes. Eradicating HIV and KP-related stigma requires transforming stigmatizing social norms and beliefs, as well as policy and legislative changes that decriminalize KP behaviors. The Population Council will continue to prioritize reducing stigma experienced by KPs and others as a central part of the HIV/AIDS response.

\section{REFERENCES}

1. Population Council.2016-2017 Integrated Biological and Behavioural Survey among Female Sex Workers in Zambia. Unpublished. Lusaka, Zambia: Population Council.

2. National MARPs Programme, NASCOP, Ministry of Health, Kenya. 2014. "2010-2011 Integrated biological and behavioural surveillance survey among key populations in Nairobi and Kisumu, Kenya." Nairobi: NASCOP, Ministry of Health, Kenya.

3. Ganju, D. and N. Saggurti. 2017. "Stigma, violence and HIV vulnerability among transgender persons in sex work in Maharashtra, India," Culture, Health \& Sexuality 19(8):903-917. doi: 10.1080/13691058.2016.1271141

4. Vu, L. et al. 2012. "Levels and correlates of internalized homophobia among men who have sex with men in Pretoria, South Africa" AIDS and Behavior 16(3): 717-723.

5. Pilgirm, N. et al. (2018, forthcoming) "Quality of care and HIV service utilization among key populations in Zambia: a qualitative comparative analysis among female sex workers, men who have sex with men and people who use drugs," AIDS and Behavior.

6. Ahonsi, B et al. 2014. "HIV/AIDS vulnerabilities, discrimination, and service accessibility among Africa's youth: Insights from a multi-country study." Abuja, Nigeria: Population Council.

7. Pulerwitz, J. et al. 2010. "Reducing HIV-related stigma: Lessons learned from Horizons research and programs," Public Health Reports 125(2): 272-281.

8. Solórzano, I. et al. 2008. "Catalyzing personal and social change around gender, sexuality, and HIV: impact evaluation of Puntos de Encuentro's communication strategy in Nicaragua" Horizons Final Report. Washington: Population Council.

9. Population Council. Unpublished. Men's Health Network Nigeria 2014 Report. Abuja, Nigeria: Population Council.

10. Population Council. 2016. "An integrated peer outreach and clinic-based intervention to improve the sexual health of young men who have sex with men in Myanmar: a Link Up evaluation," Link Up Study Brief. Washington, DC: Population Council.

11. Geibel, S. et al. 2017. "Stigma reduction training improves healthcare provider attitudes toward, and experiences of, young marginalized people in Bangladesh," Journal of Adolescent Health 60(2): S35-S44.

12. Moreau, A. et al. 2007. "Implementing STI/HIV prevention and care interventions for men who have sex with men in Senegal," Horizons Research Summary. Washington: Population Council.

13. Tun, W. et al. (2018, forthcoming) Uptake of HIV self-testing and linkage to treatment among men who have sex with men (MSM) in Nigeria: A pilot program using key opinion leaders to reach MSM, Journal pending.

14. Project SOAR. 2018. "Early results demonstrate the importance of early treatment of HIV and the feasibility and acceptability of community-based antiretroviral treatment delivery for female sex workers in Tanzania," Project SOAR Results Brief. Washington, DC: Population Council

15. Population Council. "Community-based HIV prevention, treatment, care \& support services for MSM and other key populations in Lagos," Program Brief. Lagos: Population Council.

16. Friedland, B., et al. 2018. "Measuring intersecting stigma among key populations living with HIV: implementing the People Living with HIV Stigma Index 2.0," Journal of the International AIDS Society 21(S5): e25131.
POPULATION COUNCIL

Ideas. Evidence. Impact.
The Population Council confronts critical health and development issues-from stopping the spread of HIV to improving reproductive health and ensuring that young people lead full and productive lives. Through biomedical, social science and public health research in about 50 countries, the Council works with our partners to deliver solutions that lead to more effective policies, programs, and technologies to improve lives worldwide. Established in 1952 and headquartered in New York, the Council is a nongovernmental, nonprofit organization with an international board of trustees.

Suggested citation: Population Council. 2018. "Addressing stigma and gender inequities to strengthen HIV and AIDS programming: A focus on key populations," Factsheet. Washington, DC: Population Council. 